\title{
Medial Prefrontal Cortex Is Necessary for an Appetitive Contextual Conditioned Stimulus to Promote Eating in Sated Rats
}

\author{
Gorica D. Petrovich, Cali A. Ross, Peter C. Holland, and Michela Gallagher \\ Department of Psychological and Brain Sciences, Johns Hopkins University, Baltimore, Maryland 21218
}

\begin{abstract}
Motivation plays an important role in the control of food intake. A cue that acquires motivational properties through pairings with food consumption when an animal is hungry can override satiety and promote eating in sated rats. This phenomenon of conditioned potentiation of feeding is mediated by connections between the forebrain and the lateral hypothalamic area (LHA). In a recent study using markers for cellular activation, neurons in the ventral medial prefrontal cortex (vmPFC) that project directly to the LHA were strongly engaged after exposure to a conditioned cue that stimulates eating in sated rats. Here, we examined whether those vmPFC neurons are necessary for conditioned potentiation of eating. We trained rats in a paradigm in which the context provided conditioning cues. Rats with bilateral neurotoxic lesions of vmPFC were impaired in context-enhanced food consumption in tests when the rats were sated. At the same time, vmPFC lesions did not produce changes in food consumption in the home cage or changes in body weight during training. Thus, vmPFC neurotoxic lesions produced impairment in food consumption specifically driven by conditioned motivational cues. The current findings suggest a critical role for vmPFC in the brain network that mediates control of conditioned motivation to eat perhaps by a mechanism akin to appetite or craving.
\end{abstract}

Key words: appetite; feeding; learning; motivation; pavlovian conditioning; satiation

\section{Introduction}

Food consumption is controlled not only by metabolic factors but also by signals that are not directly related to homeostasis. For example, rats that are trained to associate a cue with food consumption when hunger prevails will subsequently consume a greater amount of food when that cue is presented under conditions of satiety (Weingarten, 1983). Previously, we showed that cue-driven food consumption critically depends on an intact amygdala and amygdalohypothalamic network (Holland et al., 2002; Petrovich et al., 2002). Recently, in a study that combined detection of immediate-early gene (IEG) induction and tract tracing methods, we found that neurons in the ventral medial prefrontal cortex (vmPFC) that directly project to the lateral hypothalamus (LHA) are activated selectively by a cue that stimulates eating in sated rats (Petrovich et al., 2005a). In addition to this evidence, other anatomical studies also suggest that this region of the PFC is well positioned to communicate with both the amygdala and lateral hypothalamus (Sesack et al., 1989; Hurley et al., 1991; Risold et al., 1997; Swanson and Petrovich, 1998; Floyd et al., 2001; Gabbott et al., 2005). A strikingly similar forebrain network for motivational control of appetite in humans is emerg-

Received Nov. 17, 2006; revised May 8, 2007; accepted May 9, 2007.

This work was supported by National Institute of Mental Health Grants MH67252 (G.D.P.) and MH60179 (M.G., P.C.H.).

Correspondence should be addressed to Dr. Gorica D. Petrovich, Department of Psychological and Brain Sciences, Johns Hopkins University, 3400 North Charles Street, Baltimore, MD 21218. E-mail: petrovich@jhu.edu.

DOI:10.1523/JNEUROSCI.5001-06.2007

Copyright $\odot 2007$ Society for Neuroscience $\quad$ 0270-6474/07/276436-06\$15.00/0 ing from studies using functional imaging methods. Most notably, activations of the amygdala and medial region of the orbitofrontal cortex are seen in response to a number of different foodrelated cues (LaBar et al., 2001; Arana et al., 2003; Gottfried et al., 2003; Killgore et al., 2003; Hinton et al., 2004; Pelchat et al., 2004).

Here, we tested whether an intact vmPFC is necessary for learned potentiation of eating. We produced neurotoxic lesions in the region of the vmPFC that encompassed the ventral prelimbic, infralimbic, and medial orbitofrontal areas, designed to closely match the region of the vmPFC that was functionally activated in potentiation tests in our previous study (Petrovich et al., 2005a). Then, we trained rats in a conditioned potentiation paradigm in which a behavioral chamber and contextual cues associated with it served as a conditioned stimulus (CS). After training, we tested sated rats for food consumption in the conditioning context. We found that the vmPFC lesions abolished context-driven food consumption specifically for the food used in training and did not impact food intake in the home cage or body weight. Preliminary results from this study were presented previously (Petrovich et al., 2005b).

\section{Materials and Methods}

Subjects. The subjects were male Long-Evans rats (Charles River Laboratory, Raleigh, NC) housed in individual cages under a $12 \mathrm{~h}$ light/dark cycle (lights on at 6:00 A.M.). After arrival, rats were acclimated for 1 week in the vivarium and then given neurotoxic lesions of vmPFC $(n=$ 28 ) or sham lesions $(n=20)$. A postoperative recovery interval of $\sim 2.5$ weeks occurred before behavioral procedures.

Surgical methods. All surgeries were performed under aseptic condi- 
A

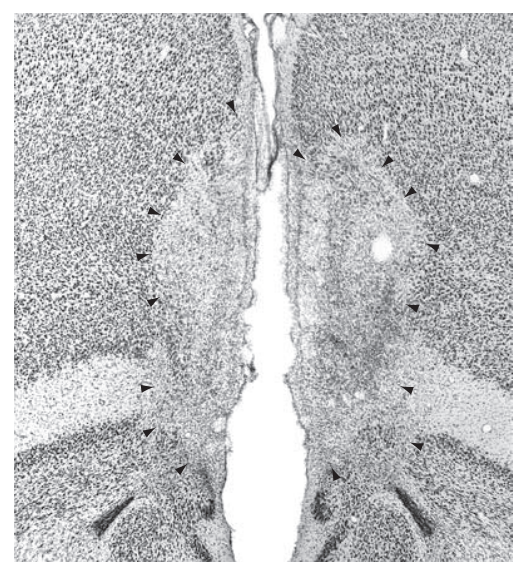

B

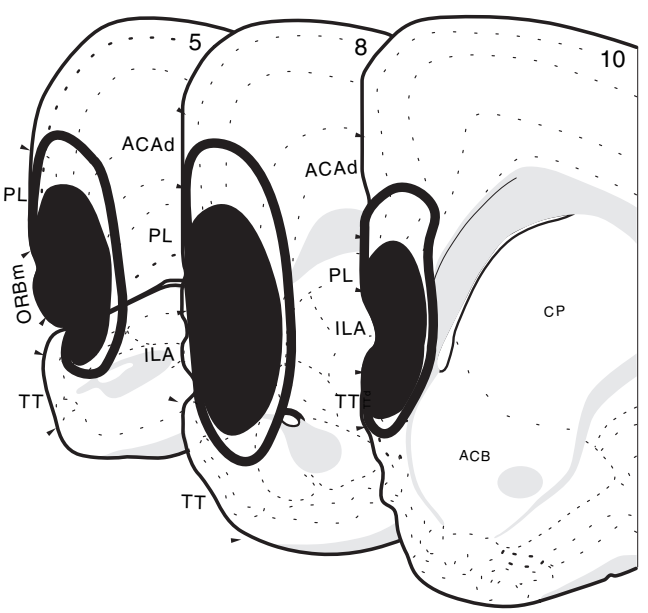

Figure 1. Histology. A, Representative photomicrographs of bilateral, vmPFC lesions. The arrowheads denote lesion borders. $\boldsymbol{B}$, The extent of the largest (enclosed black area) and smallest (filled black area) acceptable vmPFC lesions at several rostrocaudal levels. The plates were adapted from the atlas of Swanson (1998-1999). ACAd, Anterior cingulate area, dorsal part; CP, caudoputamen; ORBm, orbital area, medial part; TT, tenia tecta.

tions using isoflurane gas for induction and maintenance of anesthesia, using a stereotaxic frame (Kopf Instruments, Tujunga, CA). Neurotoxic bilateral lesions were made with $20 \mathrm{mg} / \mathrm{ml}$ NMDA (Sigma, St. Louis, $\mathrm{MO}$ ) in a $0.1 \mathrm{M}$ phosphate buffer solution. Each rat received a total of four injections (two sites in each hemisphere of $0.1 \mu \mathrm{l}$ ) using a 30 gauge needle attached by a length of plastic tubing to a $10 \mu \mathrm{l}$ microsyringe (Hamilton, Reno, NV) mounted on a syringe pump (Sage Instruments, Boston, MA). The flat-skull coordinates from bregma were as follows: anteroposterior, $3.20 \mathrm{~mm}$; mediolateral, $\pm 0.8 \mathrm{~mm}$; and dorsoventral (taken at the site of injections), $4.00 \mathrm{~mm}$ and $4.7 \mathrm{~mm}$. Sham lesions were made by infusing phosphate buffer vehicle alone. After each infusion, the needle was left in place for 4 min to allow for diffusion.

Apparatus. The behavioral training apparatus consisted of four identical chambers $(30 \times 24 \times 30 \mathrm{~cm}$; Colbourn Instruments, Allentown, PA), with aluminum top and sides and a transparent Plexiglas back and front. Dark blue Plexiglas was placed on top of the grid floor so that rats could not see or feel the grids. Each chamber contained a recessed food cup $(3.2 \times 4.2 \mathrm{~cm})$ and an opaque glass food bowl, $9 \mathrm{~cm}$ in diameter and $7 \mathrm{~cm}$ high, which was located on the opposite side of the chamber from the food cup. Dim background illumination was provided by two $25 \mathrm{~W}$ red bulbs, each placed $1.5 \mathrm{~m}$ from the chambers. Masking noise ( $60 \mathrm{~dB})$ was provided by ventilation fans located outside the conditioning chambers. The experimental chambers were wiped with $1 \%$ acetic acid solution before each animal was placed in them for training or testing sessions. Video cameras in the test chambers recorded behavior during training and testing.

Behavioral training procedure. Rats were trained in a conditioned potentiation paradigm in which the behavioral chamber served as the context that was paired with food consumption under food deprivation. Approximately $24 \mathrm{~h}$ before training, all rats were given $\sim 1 \mathrm{~g}$ of the training pellets in the home cage to familiarize them with those pellets. The training consisted of six sessions; all rats were food deprived for $19 \mathrm{~h}$ before each of those sessions. For each training session, rats were transported in the home cage from the vivarium to the behavioral testing room. Rats in the paired group were placed in the conditioning context with $7 \mathrm{~g}$ of training food pellets (formula PJPPP; $45 \mathrm{mg}$; Research Diets, New Brunswick, NJ) in the food cup. After $10 \mathrm{~min}$, rats were taken out of the conditioning context, placed into the home cage, and transported back to the vivarium. Rats in the unpaired group were placed in the same context without food for $10 \mathrm{~min}$ and then returned to the vivarium, where the training pellets were provided in the home cage after $1-3 \mathrm{~h}$ had passed from exposure to the training apparatus. The amount of the training pellets given to rats in the unpaired group was matched to the average amount eaten by the rats in the paired group in the conditioning context.
After consumption of the training pellets by rats in the unpaired group, all rats were given food chow (18\% Protein Rodent Diet; Harlan Teklad Global Diets \#2018; Harlan Teklad, Madison, WI) ad libitum for $24 \mathrm{~h}$ before they were deprived before the next training session. The rats in paired and unpaired groups were matched for the time of the day they were trained, as well as the amount of time that lapsed between training and the time food chow was returned to the home cage. The amount of food chow consumed in the home cage after each training session in a $1 \mathrm{~h}$ period was recorded. Additionally, rats that failed to increase food consumption during training were removed from the study (three shams and two lesions).

After the last training session, rats were given ad libitum access to food chow and water throughout the remainder of the behavioral protocol; testing started $2 \mathrm{~d}$ after ad libitum access to food and water was reinstated. Rats were tested in four food-consumption tests that were conducted on 4 consecutive days. For each test, rats were placed in the conditioning context for a total of $10 \mathrm{~min}$ with $15 \mathrm{~g}$ of food in the food bowl, which had been empty during training sessions. We tested food consumption in a receptacle and location other than that in which food had been delivered during training to minimize possible enhancement of food consumption as a result of conditioned food cup approach behavior. Consumption at another location in the chamber would better reflect conditioned motivational properties acquired by contextual cues. After $10 \mathrm{~min}$, the rats were taken out of the conditioning context, and any remaining food was removed and weighed. On the first and last tests, rats were tested for food consumption with the training pellets. On the second test, rats were provided with novel food pellets (formula PJAI; $45 \mathrm{mg}$; Research Diets), and on the third test, rats were tested for food consumption in the conditioning context with their standard food chow. Immediately after testing, rats were returned to the vivarium and given access to food chow and water ad libitum. The amount of food chow consumed in the home cage in the first hour after each test was also recorded.

The experiments were conducted in two replications with 24 rats in each (lesion, 14; sham, 10). In the first replication, rats were tested after four training sessions in one food-consumption test with the training pellets. Those data indicated that the training was insufficient to support robust potentiation in control rats (shams, unpaired, $1.8 \pm 0.8 \mathrm{~g}$; paired, $3.3 \pm 2.5 \mathrm{~g}$; lesion, unpaired, $1.0 \pm 0.3 \mathrm{~g}$; paired, $1.9 \pm 0.3 \mathrm{~g}$ ), and rats were given a reminder training session (sham, $5.5 \pm 1.5 \mathrm{~g}$; lesion, $5.7 \pm$ $0.8 \mathrm{~g}$ ) and then returned to complete the training schedule described above. Otherwise, the replications were identical.

Statistics. Behavioral data were analyzed using ANOVA, followed by Fisher's PLSD tests when appropriate. In all cases, $p<0.05$ was considered significant.

Histological procedure. After completion of all behavioral procedures, rats were anesthetized and perfused intracardially with $0.9 \%$ saline, followed by $4 \%$ Formalin in $0.1 \mathrm{~m}$ PBS. The brains were removed and stored in the Formalin solution used for perfusion with $12 \%$ sucrose for $48 \mathrm{~h}$. The brains were sliced on a freezing microtome, and coronal sections ( 40 $\mu \mathrm{m}$ ) were collected through the vmPFC were mounted on slides and stained with thionin. Lesion placements were verified under a light microscope and drawn onto plates adapted from rat atlas (Swanson, 1998-1999).

\section{Results}

\section{Histology}

The vmPFC lesions encompassed the prelimbic, infralimbic, and medial orbitofrontal cortical areas (Fig. $1 A, B$ ). The damage to the infralimbic region was complete, whereas only the ventral half of the prelimbic area was consistently damaged. Within the me- 


\section{Food pellet consumption in the conditioning context during training}

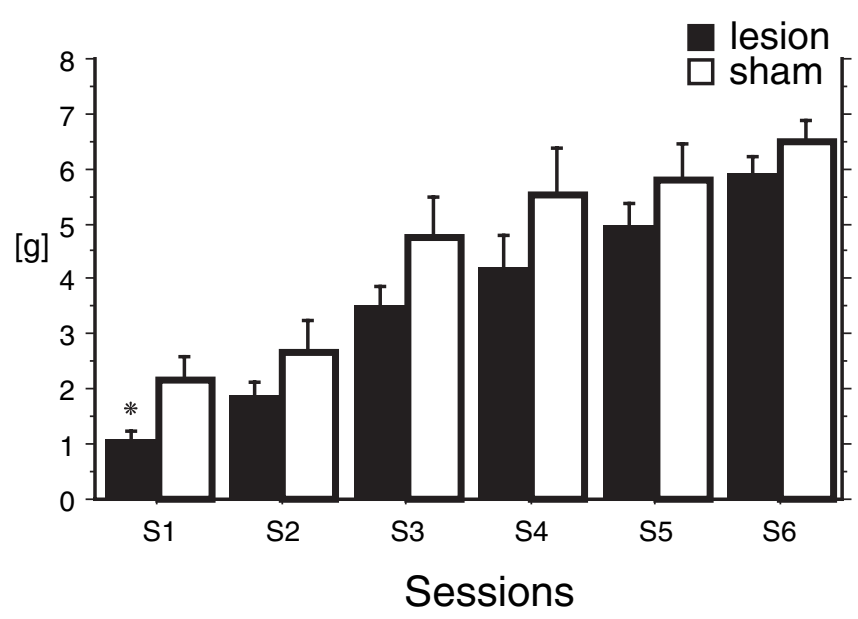

Figure 2. Food pellet consumption during training. An asterisk indicates significant difference in consumption between rats in the lesioned and sham groups $(p<0.05)$.

dial orbitofrontal cortex [mOFC; corresponds to "dorsal peduncular cortex" of Hurley et al. (1991) and Fisk and Wyss (1999)], damage was confined to the medial orbital area (as defined by Swanson, 1998-1999), with minimal damage to adjacent medial parts of the ventral and ventrolateral areas (Swanson, 1998-1999). No damage was observed within the lateral OFC. The vmPFC area lesioned here corresponds to ventral component of the medial PFC of Dalley et al. (2004). Lesions were rejected by a preestablished criterion if the overall unilateral damage to the ventral prelimbic and infralimbic regions was $<50 \%$ or if that amount of minimal damage was only achieved in one hemisphere $(n=4)$. In all acceptable lesions, there was substantial $(>75 \%)$ damage to the infralimbic, ventral half of the prelimbic areas and medial orbitofrontal cortex. No damage was observed in sham-lesioned rats.

\section{Behavioral training}

Food-restricted rats in both vmPFC- and sham-lesioned paired groups increased food consumption in the conditioning context during training (Fig. 2). With the exception of the first training session, vmPFC- and sham-lesioned rats ate a similar amount of food pellets in the conditioning context during training (Fig. 2). During the first training session only, vmPFC-lesioned rats consumed fewer pellets than sham-lesioned controls $\left(F_{(1,15)}=7.6\right.$; $p<0.02)$. The vmPFC- and sham-lesioned rats in the unpaired groups consumed all of the food pellets available in the home cage on each day. There was no difference in food chow consumption in the home cage or in body weight between vmPFC- and shamlesioned rats in the paired or unpaired groups during either training or testing (Table 1).

\section{Behavioral food-consumption tests to measure learned potentiation of feeding}

After training, rats were sated and then tested for food consumption in the conditioning context with the training pellets. In this test, sham-lesioned rats showed conditioned potentiation of eating: rats in the paired group ate more food pellets than rats in the unpaired group (Fig. 3, Test 1). In contrast, during the same test, no effect of conditioning was observed for rats with vmPFC lesions; the paired and unpaired vmPFC groups consumed similar
Table 1. Body weight and food chow consumption during training

\begin{tabular}{|c|c|c|c|c|}
\hline & \multicolumn{2}{|l|}{ Lesion } & \multicolumn{2}{|l|}{ Sham } \\
\hline & Paired & Unpaired & Paired & Unpaired \\
\hline \multicolumn{5}{|l|}{ Body weight (g) } \\
\hline Start of training & $379 \pm 7$ & $370 \pm 10$ & $391 \pm 10$ & $383 \pm 10$ \\
\hline Testing & $424 \pm 11$ & $422 \pm 10$ & $426 \pm 17$ & $428 \pm 8$ \\
\hline \multicolumn{5}{|c|}{ Food chow consumption (g) } \\
\hline After session 1 & $4.6 \pm 0.5$ & $4.4 \pm 0.5$ & $5.8 \pm 0.8$ & $4.8 \pm 0.5$ \\
\hline After session 2 & $5.2 \pm 0.4$ & $6.2 \pm 0.7$ & $5.9 \pm 0.9$ & $4.2 \pm 0.7$ \\
\hline After session 3 & $5.9 \pm 0.7$ & $4.3 \pm 0.8$ & $5.0 \pm 1.3$ & $3.9 \pm 0.4$ \\
\hline After session 4 & $5.3 \pm 0.9$ & $3.8 \pm 0.5$ & $4.2 \pm 0.9$ & $4.0 \pm 0.4$ \\
\hline After session 5 & $5.5 \pm 0.6$ & $5.2 \pm 0.6$ & $5.0 \pm 0.9$ & $4.5 \pm 0.5$ \\
\hline After session 6 & $5.1 \pm 0.6$ & $4.9 \pm 0.5$ & $4.6 \pm 0.8$ & $4.3 \pm 0.4$ \\
\hline
\end{tabular}

There was no difference in body weight (mean $\pm S E M$ ) during training and testing between rats in different groups. Food chow consumption during $60 \mathrm{~min}$ (mean \pm SEM) in home cages $2-4 \mathrm{~h}$ after each training session was similar for all rats.

\section{Food consumption in the conditioning context during tests}

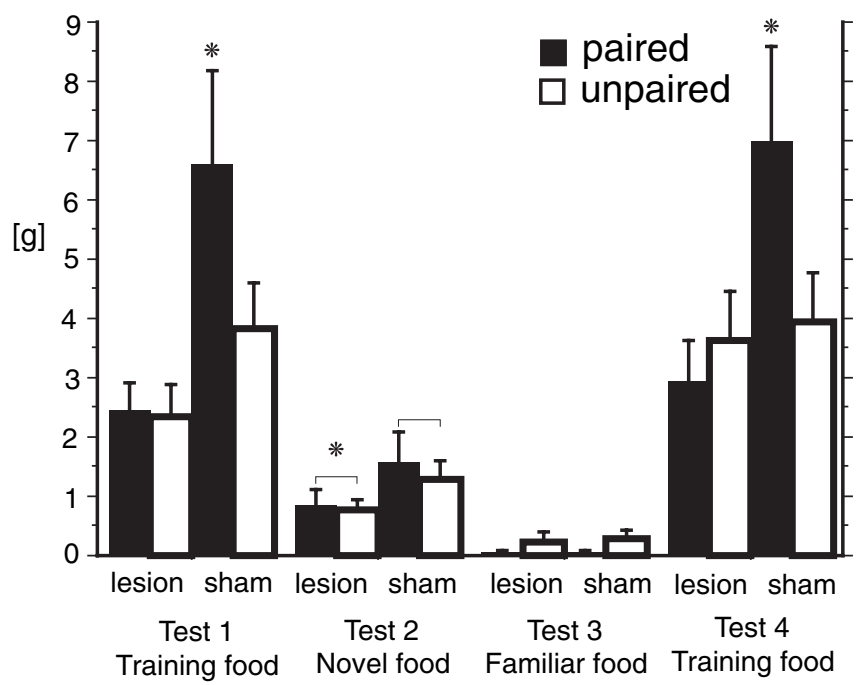

Figure 3. Food consumption (mean $\pm S E M$ ) in the conditioning context during tests. An asterisk indicates significant difference in consumption $(p<0.05)$. See Results for details.

amounts of food pellets (Fig. 3, Test 1). This description of the data is supported statistically. An ANOVA of the pellet consumption in the first consumption test with lesion type (lesion or sham) and training condition (paired or unpaired) as factors revealed main effects of lesion $\left(F_{(1,35)}=11.5 ; p<0.002\right)$. Subsequent comparisons showed greater consumption by shamlesioned rats in the paired group relative to the unpaired group $(p<0.05)$, whereas there was no difference in consumption by rats with vmPFC lesions in paired and unpaired groups $(p=$ 0.92). Furthermore, vmPFC-lesioned rats in the paired group ate significantly less than sham-lesioned rats in the paired group $(p<0.003)$. Consumption of unpaired vmPFC-lesioned rats was not significantly different from consumption of unpaired shams $(p=0.18)$. We also measured food cup entries during the first $10 \mathrm{~s}$ of the test and found no difference between rats with vmPFC lesions and sham-lesioned rats; rats in paired groups spent more time in the food cup than rats in unpaired groups (vmPFC, paired, $12.5 \pm 3 \%$; unpaired, $0 \%$; controls, paired, $14 \pm 2 \%$; unpaired, $1.2 \pm 1 \%)$. Note that in the consumption tests, the food was placed in bowls, and so the food cups (used in training) were empty. These data show that the learned tendency to ap- 
proach the food cup in paired rats did not differ between shamand vmPFC-lesioned rats and thus did not contribute to lesionrelated differences in eating from the bowls.

After the first test, rats were assessed in additional tests to determine whether the acquisition of potentiated consumption was specific to the training pellets. Rats were tested for consumption of a novel food (Fig. 3, Test 2) and then consumption of a familiar (nontraining) food (Fig. 3, Test 3) in the training context. No CS-potentiated eating was observed in the tests with nontraining pellets, regardless of whether the food was novel $\left(F_{(1,35)}=1.70 ; p=0.20\right)$ or familiar $\left(F_{(1,35)}=0.70 ; p=0.41\right)$. Interestingly, in food-consumption tests with novel pellets (Fig. 3 , Test 2$)$, there was an overall lesion effect $\left(F_{(1,35)}=4.79 ; p<\right.$ $0.05)$, attributable to a decrease in consumption by vmPFClesioned rats compared with consumption of the shams. This suppression of consumption by the vmPFC-lesioned rats in the test with novel food is similar to suppression of the vmPFClesioned rats in the first training session in a novel environment (Fig. 2). In contrast, in the test with familiar but nontraining food, consumption was similar for vmPFC-lesioned and shamlesioned rats $\left(F_{(1,35)}=0.149 ; p=0.70\right)$. Finally, the pattern of consumption of the training pellets in a fourth test was similar to the results obtained in the first test. Only sham-lesioned controls showed potentiation of eating. There was a main effect of lesion $\left(F_{(1,35)}=5.1 ; p<0.03\right)$, and the lesion by pairing interaction was close to significance $\left(F_{(1,35)}=3.7 ; p=0.061\right)$. Follow-up comparisons showed that pellet consumption of the sham-lesioned rats in the paired group differed from the consumption of the sham-lesioned rats in the unpaired $(p<0.05)$ group, as well as from consumption of the lesioned rats in the paired $(p<0.01)$ and unpaired $(p<0.05)$ groups.

\section{Discussion}

Our main finding is that vmPFC damage impairs learned enhancement of food consumption. Bilateral neurotoxic lesions of the vmPFC produced a selective impairment in food consumption driven by contextual conditioned cues.

The vmPFC area here is well matched to the ventral subregion of the medial PFC of Dalley et al. (2004), which was defined based on anatomical and functional evidence. In the current study, lesions were centered in the infralimbic area (ILA) with additional damage to the ventral prelimbic (PL) and medial OFC cortical areas. There was no damage to the lateral OFC, and only in the largest acceptable lesions did damage extend into the dorsal PL. This distinction is important because anatomical evidence suggests similarities between the ventral PL and ILA and dorsal PL and cingulate cortical areas (Sesack et al., 1989; Hurley et al., 1991; Fisk and Wyss, 1999). Similarly, the mOFC (corresponds to dorsal peduncular cortex) is strikingly similar to the ILA and distinct from lateral OFC (Sesack et al., 1989; Hurley et al., 1991; Floyd et al., 2001; Petrovich et al., 2005a). In addition, the ILA shares bidirectional connections with both PL and mOFC, whereas the latter two are not interconnected (Fisk and Wyss, 1999). Thus, the functional impairment in the current study could be caused by damage to the ILA alone or might be a consequence of disruption of the system formed by the three regions.

Additional connectional features justify grouping the vmPFC subregions. This area of the MPFC is connected with the hippocampal formation (Swanson et al., 1987; Van Groen and Wyss, 1990; Condé et al., 1995), relevant for contextual information, and its integration in motivational processing associated with appetitive learning and the control of ingestive behavior (Ito et al., 2005; Davidson et al., 2005). Similarly, the ILA, ventral (but not dorsal) PL, and medial (but not lateral) OFC all send output to the nucleus accumbens (ACB) (Sesack et al., 1989; Hurley et al., 1991; Petrovich et al., 2005a), which, itself, has been heavily implicated in feeding behavior (Baldo and Kelley, 2007). Thus, our lesions are likely to substantially eliminate direct $\mathrm{mPFC}$ efferents to the ACB, which might be important for effects on food neophobia and CS-driven eating observed here. Interestingly, a preparation that disconnected communication between the ACB and basolateral area of the amygdala did not abolish CSenhanced eating (Holland and Petrovich, 2005). Similarly, ACB neurons that project to the lateral hypothalamus did not show selective IEG induction during consumption tests with the CS (Petrovich et al., 2005a). Nevertheless, it is interesting to consider the possibility that the ACB contributes to CS-driven consumption or food neophobia via its communication with the vmPFC.

Enhanced food consumption by the contextual CS shown here is in agreement with previous results showing CS enhancement of eating with explicit cues (Weingarten, 1983; Birch et al., 1989; Holland et al., 2002; Petrovich et al., 2002, 2005a) and is similar to context-enhanced eating recently shown in intact rats (Petrovich et al., 2007) and mice (Le Merrer and Stephens, 2006). The contextual CS enhancement of food consumption is attributable to associative learning; all rats had equal exposure to both the context and pellets, but only the rats that were fed the pellets in the context during training show enhanced consumption in the tests. Also, the learned context-induced eating is not simply a consequence of the animals learning to approach the food cup, because during tests the foods were presented in a food receptacle that was different in appearance and location from the food cup used in training. Thus, learned enhancement of food consumption is a consequence of motivational properties acquired through pavlovian conditioning.

The current study examined the contribution of the vmPFC to the enhancement of eating by conditioned contextual cues. Thus, the current data do not necessarily address whether this region is also critical for control of food consumption by discrete CSs. However, in our study that combined tract tracing with IEG induction (Petrovich et al., 2005a), neurons within the vmPFC were activated by the discrete $\mathrm{CS}+$ relative to $\mathrm{CS}-$ in the same context. Thus, the current results together with our previous findings (Petrovich et al., 2002, 2005a; Holland and Petrovich, 2005) suggest that the vmPFC is a critical part of the forebrain network formed by the basolateral amygdala and LHA that mediates CS-enhanced eating by contextual and also by explicit CSs.

The current results and another recent behavioral study using similar procedures (Petrovich et al., 2007) suggest that the CSenhanced eating is food specific, and as such likely involves induction of a relatively specific motivational state, perhaps akin to appetite or craving, rather than induction of a more general motivational state, akin to hunger. Although there is no consensus definition of food cravings, especially in animal models (Weingarten and Elston, 1990), cravings for food in humans can be elicited by exposure to food cues and are often associated with binge eating (Fedoroff et al., 1997; Jansen, 1998; Sobik et al., 2005; Tiggemann and Kemps, 2005). It is particularly notable that food cues activate the medial region of the OFC in humans (Arana et al., 2003; Hinton et al., 2004). Moreover, similar regions of the PFC may also participate in the brain networks mediating cueinduced cravings for drugs. The OFC, including its medial regions, is activated by cue-induced cigarette cravings in humans (Brody et al., 2002). Similarly, regions overlapping the vmPFC targeted in the current investigation are also activated by chocolate- and nicotine-associated contextual cues in rats (Schr- 
oeder et al., 2001). Although additional studies are needed to indicate whether this rat model parallels the role of human medial OFC in appetite and cravings, the current results provide evidence for a functional dissociation within the OFC in the rodent brain. The impairment in the current study is linked to the medial OFC, whereas previous findings showed that the lateral OFC is not necessary for CS-enhanced food consumption (McDannald et al., 2005). Thus, substantial evidence suggests that the ventral areas within the rat vmPFC (approximately the medial regions of the OFC) could represent a functional counterpart for the medial OFC in humans (Öngür and Price, 2000).

The motivation to eat driven by the CS may also involve overriding sensory-specific satiety (Rolls et al., 1981). As reported previously, food cues increase dopamine efflux within the medial PFC in both food-deprived (Ahn and Phillips, 1999) and nondeprived (Bassareo and Di Chiara, 1997) rats. And importantly, dopamine efflux within the vmPFC was correlated with decreased consumption of palatable foods as a result of sensoryspecific satiety. Likewise, activation of the human OFC decreased selectively in response to an olfactory cue of a food eaten to satiety but not to an odor of a food not eaten (O'Doherty et al., 2000). Also, a decrease in regional cerebral blood flow within the human caudomedial OFC is shown as subjects' rating changes from pleasant to aversive in the course of chocolate satiation experiment (Small et al., 2001). Still, the specific mechanisms by which food-associated cues stimulate eating in sated states, by increasing craving, decreasing satiety, or a combination of such processes, remain to be elucidated.

In addition to eating under the influence of learned cues, the vmPFC contributes to modulation of food intake by novelty. Here, we found that vmPFC-lesioned rats ate less than controls when food was presented in a novel environment or when a novel food was first presented in a familiar environment. This impairment is likely an exaggeration of neophobia, a species-typical adaptive response to novel food, and suggests a role for intact vmPFC in regulation of unlearned adaptive feeding responses.

A previous study also showed effects of lesions of the ventral medial PFC on food neophobia (Burns et al., 1996). In that study, rats with neurotoxic lesions of the PL were given a choice between familiar lab chow and a novel, preferred food in an unfamiliar, open field environment. Rats with lesions showed an increased trend to taste novel food before tasting familiar food and an overall increase in latency to eat familiar food, compared with shams. However, the amount consumed of the two foods was similar for rats with lesions and controls. Thus, Burns et al. (1996) found reduced, whereas we find exaggerated, food neophobia in rats with $\mathrm{mPFC}$ lesions. In that regard, lesions of Burns et al. (1996) were centered in the PL, whereas our lesions were centered in the ILA (with damage to the adjacent ventral PL and mOFC) regions. Thus, the findings of the two studies together might suggest different roles for two of the subregions within the vmPFC in food neophobia. However, there are also important procedural differences between Burns et al. (1996) and our study that could have contributed to the different findings, including differences in food deprivation, testing environment, and whether one or more foods were present during tests.

Together, the impairments after vmPFC damage noted here suggest a broader function of the vmPFC in control of motivation to eat under nonmetabolic influences. Viewed within its well known role in goal-directed behavior (O'Doherty, 2004), the vmPFC appears critically involved in controlling an impulse to eat according to environmental cues. In turn, dysfunction of the vmPFC, and its associated brain network, could provide a model for features of feeding in humans relevant to overeating, appetite, and cravings.

\section{References}

Ahn S, Phillips AG (1999) Dopaminergic correlates of sensory-specific satiety in the medial prefrontal cortex and nucleus accumbens of the rat. J Neurosci 19:RC29(1-6).

Arana FS, Parkinson JA, Hinton E, Holland AJ, Owen AM, Roberts AC (2003) Dissociable contributions of the human amygdala and orbitofrontal cortex to incentive motivation and goal selection. J Neurosci 23:9632-9638.

Baldo BA, Kelley AE (2007) Discrete neurochemical coding of distinguishable motivational processes: insights from nucleus accumbens control of feeding. Psychopharmacology 191:439-459.

Bassareo V, Di Chiara G (1997) Differential influence of associative and nonassociative learning mechanisms on the responsiveness of prefrontal and accumbal dopamine transmission to food stimuli in rats fed ad libitum. J Neurosci 15:851-861.

Birch LL, McPhee L, Sullivan S, Johnson S (1989) Conditioned meal initiation in young children. Appetite 13:105-113.

Brody AL, Mandelkern MA, London ED, Childress AR, Lee GS, Bota RG, Ho ML, Saxena S, Baxter LRJ, Madsen D, Jarvik ME (2002) Brain metabolic changes during cigarette craving. Arch Gen Psychiatry 59:1162-1172.

Burns LH, Annett L, Kelley AE, Everitt BJ, Robbins TW (1996) Effects of lesions to amygdala, ventral subiculum, medial prefrontal cortex, and nucleus accumbens on the reaction to novelty: implication for limbicstriatal interactions. Behav Neurosci 110:60-73.

Condé F, Maire-Lepoivre E, Audinat E, Crépel F (1995) Afferent connections of the medial frontal cortex of the rat. II. Cortical and subcortical afferents. J Comp Neurol 352:567-593.

Dalley JW, Cardinal RN, Robbins TW (2004) Prefrontal executive and cognitive functions in rodents: neural and neurochemical substrates. Neurosci Biobehav Rev 28:771-784.

Davidson TL, Kanoski SE, Walls EK, Jarrard LE (2005) Memory inhibition and energy regulation. Physiol Behav 86:731-746.

Fedoroff IC, Polivy J, Herman CP (1997) The effect of pre-exposure to food cues on the eating behavior of restrained and unrestrained eaters. Appetite 28:33-47.

Fisk GD, Wyss JM (1999) Associational projections of the anterior midline cortex in the rat: intracingulate and retrosplenial connections. Brain Res $825: 1-13$.

Floyd NS, Price JL, Ferry AT, Keay KA, Bandler R (2001) Orbitomedial prefrontal cortical projections to hypothalamus in the rat. J Comp Neurol 432:307-328.

Gabbott PL, Warner TA, Jays PR, Salway P, Busby SJ (2005) Prefrontal cortex in the rat: projections to subcortical autonomic, motor, and limbic centers. J Comp Neurol 492:145-177.

Gottfried JA, O'Doherty J, Dolan RJ (2003) Encoding predictive reward value in human amygdala and orbitofrontal cortex. Science 301:1104-1107.

Hinton EC, Parkinson JA, Holland AJ, Arana FS, Roberts AC, Owen AM (2004) Neural contributions to the motivational control of appetite in humans. Eur J Neurosci 20:1411-1418.

Holland PC, Petrovich GD (2005) A neural systems analysis of the potentiation of feeding by conditioned stimuli. Physiol Behav 86:747-761.

Holland PC, Petrovich GD, Gallagher M (2002) The effects of amygdala lesions on conditioned stimulus-potentiated eating in rats. Physiol Behav 76:117-129.

Hurley KM, Herbert H, Moga MM, Saper CB (1991) Efferent projections of the infralimbic cortex of the rat. J Comp Neurol 308:249-276.

Ito R, Everitt BJ, Robbins TW (2005) The hippocampus and appetitive pavlovian conditioning: effects of excitotoxic hippocampal lesions on conditioned locomotor activity and autoshaping. Hippocampus 15:713-721.

Jansen A (1998) A learning model of binge eating: cue reactivity and cue exposure. Behav Res Ther 36:257-272.

Killgore WDS, Young AD, Femia LA, Bogorodzki P, Rogowska J, YurgelunTodd DA (2003) Cortical and limbic activation during viewing of highversus low-calorie foods. NeuroImage 19:1381-1394.

LaBar KS, Gitelman DR, Parrish TB, Kim YH, Nobre AC, Mesulam MM (2001) Hunger selectively modulates corticolimbic activation to food stimuli in humans. Behav Neurosci 115:493-500.

Le Merrer J, Stephens DN (2006) Food-induced behavioral sensitization, its 
cross-sensitization to cocaine and morphine, pharmacological blockade, and effect on food intake. J Neurosci 26:7163-7171.

McDannald MA, Saddoris MP, Gallagher M, Holland PC (2005) Lesions of orbitofrontal cortex impair rats' differential outcome expectancy learning but not conditioned stimulus-potentiated feeding. J Neurosci 25:4626-4632.

O'Doherty J (2004) Reward representations and reward-related learning in the human brain: insights from neuroimaging. Curr Opin Neurobiol 14:769-776.

O’Doherty J, Rolls ET, Francis S, Bowtell R, McGlone F, Kobal G, Renner B, Ahne G (2000) Sensory-specific satiety-related olfactory activation of the human orbitofrontal cortex. NeuroReport 11:893-897.

Öngür D, Price JL (2000) The organization of networks within the orbital and medial prefrontal cortex of rats, monkeys and humans. Cereb Cortex 10:206-219.

Pelchat ML, Johnson A, Chan R, Valdez J, Ragland JD (2004) Images of desire: food-craving activation during fMRI. NeuroImage 23:1486-1493.

Petrovich GD, Setlow B, Holland PC, Gallagher M (2002) Amygdalohypothalamic circuit allows learned cues to override satiety and promote eating. J Neurosci 22:8748-8753.

Petrovich GD, Holland PC, Gallagher M (2005a) Amygdalar and prefrontal pathways to the lateral hypothalamus are activated by a learned cue that stimulates eating. J Neurosci 25:8295-8302.

Petrovich GD, Holland PC, Gallagher M (2005b) Medial prefrontal cortex is necessary for an appetitive conditioned stimulus to promote eating in sated rats. Soc Neurosci Abstr 31:658.8.

Petrovich GD, Ross CA, Gallagher M, Holland PC (2007) Learned contextual cue potentiates eating in rats. Physiol Behav 90:362-367.

Risold PY, Thompson RH, Swanson LW (1997) The structural organization of connections between hypothalamus and cerebral cortex. Brain Res Rev 24:197-254
Rolls BJ, Rolls ET, Rowe EA, Sweeney K (1981) Sensory specific satiety in man. Physiol Behav 27:137-142.

Schroeder BE, Binzak JM, Kelley AE (2001) A common profile of prefrontal cortical activation following exposure to nicotine- or chocolateassociated contextual cues. Neuroscience 105:535-545.

Sesack SR, Deutch AY, Roth RH, Bunney BS (1989) Topographical organization of the efferent projections of the medial prefrontal cortex in the rat: an anterograde tract-tracing study with Phaseolus vulgaris leucoagglutinin. J Comp Neurol 290:213-242.

Small DM, Zatorre RJ, Dagher A, Evans AC, Jones-Gotman M (2001) Changes in brain activity related to eating chocolate: from pleasure to aversion. Brain 124:1720-1733.

Sobik L, Hutchison K, Craighead L (2005) Cue-elicited craving for food: a fresh approach to the study of binge eating. Appetite 44:253-261.

Swanson LW (1998-1999) Brain maps: structure of the rat brain, Ed 2. Amsterdam: Elsevier.

Swanson LW, Petrovich GD (1998) What is the amygdala? Trends Neurosci 21:323-331.

Swanson LW, Köhler C, Björklund A (1987) The limbic region. I: The septohippocampal system. In: Handbook of chemical neuroanatomy, Vol 5, Integrated systems of the CNS, Part I. (Björklund A, Hökfelt T, Swanson LW, eds), pp 125-277. Amsterdam: Elsevier.

Tiggemann M, Kemps E (2005) The phenomenology of food cravings: the role of mental imagery. Appetite 45:305-313.

Van Groen T, Wyss JM (1990) Extrinsic projections from area CA1 of the hippocampus: olfactory, cortical, subcortical, and bilateral hippocampal formation projections. J Comp Neurol 302:515-528.

Weingarten HP (1983) Conditioned cues elicit feeding in sated rats: a role for learning in meal initiation. Science 220:431-433.

Weingarten HP, Elston D (1990) The phenomenology of food craving. Appetite 15:231-246. 